\title{
Language Network Function in Young Children Born Very Preterm
}

\author{
Eun Jung Choi ${ }^{1,2 *}$, Marlee M. Vandewouw ${ }^{1,3}$, Julia M. Young ${ }^{1,3,4}$ and Margot J. Taylor 1,3,4,5 \\ ${ }^{1}$ Department of Diagnostic Imaging, The Hospital for Sick Children, Toronto, ON, Canada, ${ }^{2}$ Bloorview Research Institute, \\ Holland Bloorview Kids Rehabilitation Hospital, Toronto, ON, Canada, ${ }^{3}$ Neurosciences \& Mental Health, SickKids Research \\ Institute, Toronto, ON, Canada, ${ }^{4}$ Department of Psychology, University of Toronto, Toronto, ON, Canada, ${ }^{5}$ Department \\ of Medical Imaging, University of Toronto, Toronto, ON, Canada
}

\section{OPEN ACCESS}

Edited by:

Peter Sörös,

University of Oldenburg, Germany

Reviewed by:

Dustin Scheinost,

Yale University, United States

Charlotte Herzmann,

University of Bremen, Germany

*Correspondence:

Eun Jung Choi

eunjung.choi@sickkids.ca

Received: 16 August 2018 Accepted: 05 December 2018 Published: 20 December 2018

Citation:

Choi EJ, Vandewouw MM, Young JM and Taylor MJ (2018) Language Network Function in Young

Children Born Very Preterm. Front. Hum. Neurosci. 12:512. doi: 10.3389/fnhum.2018.00512
Language deficits are reported in preterm born children across development. Recent neuroimaging studies have found functional alterations in large-scale brain networks underlying these language deficits, but the early childhood development of the language network has not been investigated. Here, we compared intrinsic language network connectivity in 4-year-old children born VPT and term-born controls, using defined language regions (Broca's area, Wernicke's areas, and their homologues in the right hemisphere). Resting-state functional magnetic resonance imaging (fMRI) was obtained, and the group differences in whole-brain connectivity were examined from each seed as well as correlations with language outcomes. We found significantly decreased functional connectivity in almost all language regions in children born VPT compared to their term controls. Notably, Broca's area homologue in the right hemisphere emerged as a functional hub of decreased connectivity in VPT group, specifically to bilateral inferior frontal and supramarginal gyri; connectivity strength between Broca's area homologue with the right supramarginal and the left inferior frontal gyri was associated with better language outcomes at 4 years of age. Wernicke's area and its homologue also showed decreased inter-hemispheric connections to bilateral supramarginal gyri in the VPT group. Decreased intra- and inter-hemispheric connectivity among primary language regions suggests immature and altered function in the language network in children born VPT.

Keywords: very preterm-born, resting-state functional connectivity, language network, Broca's area, Wernicke's area, children

\section{INTRODUCTION}

Every year, an estimated 15 million babies are born preterm and this number is rising (World Health Organization [WHO], 2018). At birth, preterm neonates suffer both major (8\%) and minor (37\%) medical morbidities (Manuck et al., 2016), but significant neurocognitive deficits are reported in childhood even among children who show no obvious neurological injury (Isaacs et al., 2004; Aarnoudse-Moens et al., 2012). In the language domain, preterm birth has been found to increase the risk of deficits in the preschool and school years (Sansavini et al., 2010; Gonzalez-Gomez and Nazzi, 2012). Poor language outcomes emerge as early as the first 2 years of life and are observed across development (Vohr, 2014). Some studies reported that language problems in pretermborn children may improve with age (Luu et al., 2009; Putnick et al., 2017). Others suggested, however, these early impairments in language do not improve, even though preterm infants 
experience an earlier and richer exposure to language (Peña et al., 2010,2012), suggesting significant neurobiological changes in the brains of children born preterm. The risk for language difficulties increases with lower gestational age such that children born very preterm (VPT, $<32$ weeks of gestational age) experience significantly lower acquisition of vocabulary at 2 years of age, and lower scores in receptive and expressive language skills and phonological perception at school age (Foster-Cohen et al., 2007; Sansavini et al., 2010; Barre et al., 2011). In addition, a meta-analysis found that these language deficits increased between 3 and 12 years of age (van Noort-van der Spek et al., 2012). However, neurobiological mechanisms underlying these difficulties are still unknown.

Structural and functional alterations in the VPT brain have been reported as the underlying neural mechanisms that mediate neurocognitive deficits. Structural alterations may be due to disturbances in the considerable neural development, including myelination, proliferation, and synaptic organization, that occurs in the last trimester of pregnancy (Counsell et al., 2003), the period of very preterm birth. Individuals born VPT have shown reduced volumes in cortical, subcortical gray, and white matter as well as the cerebellum, compared to term-born controls (Parker et al., 2008; Carreiras et al., 2009; Ment et al., 2009; Young et al., 2015, 2018). Of note, results of volumetric and microstructural connectivity changes in language areas, including the left frontal regions, temporal, and parietal cortices, have been implicated in poor language function in this population (Lind et al., 2011; Nosarti et al., 2011).

Recent advances in functional neuroimaging techniques have highlighted resting-state functional connectivity, which measures the temporal correlations of blood-oxygen-leveldependent (BOLD) signal among spatially distributed brain regions; regions with correlated activity are believed to form intrinsic functional networks (Friston et al., 1996). This approach towards a systematic understanding of large-scale functional brain organization has had huge benefits in expanding our knowledge of neural mechanisms of various clinical conditions. A recent study, which reviewed atypical functional connectivity in individuals born preterm across development, suggested longlasting changes in language organization from the fetal period to young adulthood (Kwon et al., 2016; Thomason et al., 2017). They showed that preterm infants demonstrated reduced connectivity in the language network compared to their term-born controls; less complexity at 38 weeks of gestational age (Smyser et al., 2014), decreased lateralization in left frontal-temporal language area, and no significant connectivity between left and right Broca's area at 42 weeks of age (Kwon et al., 2015). However, other studies have found that older children and adolescents had evidence of both increased (Gozzo et al., 2009) and decreased connectivity in the language network (Scheinost et al., 2014). The functional alterations of language-related brain areas were also reported in young adulthood (Scheinost et al., 2012; Constable et al., 2013; Bäuml et al., 2014). Although converging evidence indicates an altered functional language network in individuals born preterm, we still lack knowledge on the effect of preterm birth on the language network development throughout the lifespan, with inconsistent results as well as significant gaps in early childhood.
Language ability develops very quickly during childhood (Berk, 2013), but the underlying neural bases are just starting to be investigated at a large-scale brain network level in young children. A recent study explored the developmental changes in intrinsic language network development in typically developing preschool children (Xiao et al., 2016a). They found that children at age 3 showed relatively strong contralateral connections in primary language regions compared to children at age 5 . In addition, the developmental changes were characterized by increasing long-range connections between left inferior frontal gyrus and superior temporal gyrus and an increase in leftward lateralization. This result corresponds to typical language network maturation from inter- to intra-hemispheric connectivity (Friederici et al., 2011), with top-down processing centered in the inferior frontal cortex starting at this age (Skeide and Friederici, 2016), and an increase of leftward lateralization in Broca's area (Tomasi and Volkow, 2012; Zhu et al., 2014). Given these behavioral and neural changes, children born preterm may have a different language network in early childhood compared to age-matched term-born children, contributing to their language difficulties; however, their intrinsic language network has not yet been examined.

We investigated the intrinsic language network across defined regions in children born VPT at 4 years of age compared to termborn controls, using resting-state functional magnetic resonance imaging (fMRI). We hypothesized altered connectivity in this network in children born VPT, specifically in primary language regions: Broca's area, Wernicke's area, and their homologues in the right hemisphere. Given the developmental pattern of maturation in older VPT children, we expected children born VPT would show decreased interhemispheric connectivity across the language regions compared to term-born children, and that the altered functional connectivity in VPT group may be associated with poorer language outcomes.

\section{MATERIALS AND METHODS}

\section{Participants}

Forty-one children born very preterm [VPT: less than 32 weeks of gestational age (GA) (mean 28 weeks of GA)] participated in this study at 4 years of age (chronological age mean $4.2 \pm 0.2$ years) (Table 1). These children were part of a longitudinal investigation of children born VPT, followed every 2 years from birth. Thirtytwo full-term (FT) born children were also recruited as controls (age mean $4.5 \pm 0.4$ years), through advertisements placed in the community, local schools, and at the hospital. Although all of the children in both groups were between 4 and 5 years of age, the term-born children were older by a few months than children born very preterm $(t=-3.393, p<0.01)$, so age was included as a covariate in all subsequent analyses. Exclusion criteria for both groups included a history of neurological or neurodevelopmental disorders, an IQ $\leq 70$, and any language or vision issues preventing successful completion of tasks as well as standard MRI exclusions. All children gave verbal assent and parents gave written informed consent. This study was approved by the Research Ethics Board at the Hospital for Sick Children. 
TABLE 1 | Demographics and head motions of children born VPT and FT in final analysis.

\begin{tabular}{lccc}
\hline & & VPT & FT \\
\hline Sample size & $n$ & 31 & 23 \\
Age (years) & Mean \pm SD & $4.2 \pm 0.2$ & $4.5 \pm 0.4$ \\
Sex & M:F & $12: 19$ & $13: 10$ \\
Gestational age (weeks) & Mean \pm SD & $28.3 \pm 1.7$ & $40 \pm 1.5$ \\
Excluded volumes (out of 120) & Mean \pm SD & $1.61 \pm 4.51$ & $1.52 \pm 2.71$ \\
Mean maximum displacement & Mean \pm SD & $0.25 \pm 0.31$ & $0.29 \pm 0.20$ \\
in head motions & & & \\
\hline
\end{tabular}

\section{Neuropsychological Assessments}

A measure of IQ was obtained for all 54 children using the Wechsler Preschool and Primary Scales of Intelligence - Third Edition (WPPSI-III; Wechsler, 2002), which included the Verbal (VIQ), Performance (PIQ), Processing Speed (PSQ), and FullScale IQ (FSIQ). Children's language outcomes were assessed by Clinical Evaluation of Language Fundamentals (CELF Pre-2; Wiig et al., 2004), which included a Core Language score (CL), Receptive Language index (RL), Expressive Language index (EL), Language Content Index (LC), and Language Structure index (LS). Visual motor integration was also assessed using the BeeryBuktenica Test of Visual Motor Integration (VMI) (Beery et al., 2004).

\section{Data Acquisition}

MRI data were acquired on a Siemens 3T Trio. A high resolution T1-weighted anatomical image was acquired $\left[\mathrm{TR} / \mathrm{TE}=2300 / 2.96 \mathrm{~ms} ; \mathrm{FA}=9^{\circ} ; \mathrm{FOV}=192 \mathrm{~mm} \times 240 \mathrm{~mm} \times\right.$ $256 \mathrm{~mm} ; 1 \mathrm{~mm}$ isotropic voxels; $5 \mathrm{~min}$ acquisition time]. Resting state functional images were obtained with a blood oxygenation level dependent (BOLD) contrast gradient echo planar imaging pulse sequence $\left[\mathrm{TR} / \mathrm{TE}=2340 / 30 \mathrm{~ms}\right.$; FA $70^{\circ}$; FOV, matrix size $=64 \times 64 \times 40 ; 3.5 \mathrm{~mm}$ isotropic voxels; 120 volumes; $5 \mathrm{~min}$ acquisition time]. The children watched a movie of their choice during the structural images through MRI-compatible goggles, and the movie was replaced with a fixation cross inside a circle centred on the screen for the resting state scan.

\section{Data Preprocessing}

All fMRI data were preprocessed in a locally developed pipeline using standard $\mathrm{AFNI}^{1}$ and $\mathrm{FSL}^{2}$ tools. The first four volumes at the beginning of the time series were dropped as scanner stabilization, leaving 116 volumes for analysis. Images were slicetime and motion corrected. We estimated spatial deviations between the reference functional image and other images using each of six motion parameters. The deviation for each image was calculated and output into a motion file, which then was used to censor timepoints that contained too much motion. Volumes in excess of $2 \mathrm{~mm}$ from the maximum mean displacement were discarded, and children who had more than $1 / 3$ of their volumes removed were excluded from the analysis. The original

${ }^{1}$ https://afni.nimh.nih.gov

${ }^{2}$ https://www.fmrib.ox.ac.uk/fsl sample included 73 children $(\mathrm{VPT}=41, \mathrm{FT}=32)$, but 19 were excluded due to motion, leaving the final sample of 54 $(\mathrm{VPT}=31, \mathrm{FT}=23)$ for analyses. When we examined the number of excluded volumes and the mean displacement of volumes for the remaining participants, there were no significant group differences in the number of excluded volumes and the mean maximum displacement (Table 1). The data were spatially smoothed using a 7-mm FWHM Gaussian kernel and bandpass temporal filtered $(0.01-0.2 \mathrm{~Hz})$ to remove magnetic field drifts of the scanner and minimize the physiological noise of high frequency components. Nuisance signals from white matter, cerebrospinal fluid, and head motion were regressed from the data.

\section{Data Analysis}

We used a standard seed-voxel correlation approach to compare the functional connectivity of the language network between the two groups of children. Two cortical voxels that represent the primary language regions and their homologues in the right hemisphere were selected and used to create each subject's ROIs (taken from Tomasi and Volkow, 2012); a voxel near the center of mass of the left pars triangularis (BA45; $-51,27$, $18 \mathrm{~mm}$; MNI coordinates), was selected to represent Broca's area (Broca L), and its homologue in the right hemisphere (51, 27, $18 \mathrm{~mm}$; MNI coordinates; Broca R). A voxel, in the left supramarginal gyrus (at the boundaries of BA39, BA4040, and BA20; $-51,-51,30 \mathrm{~mm}$; MNI coordinates), was selected to represent Wernicke's area (Wernicke L), and its homologue in the right hemisphere $(51,-51,30 \mathrm{~mm}$; MNI coordinates; Wernicke $\mathrm{R})$. To account for inter-subject variability, the seed coordinates were dilated within a $10-\mathrm{mm}$ spherical ROI until the internal correlation dropped below 0.7 or the number of voxels exceeded 300 (see Supplement 1 for more details). First-level analysis was performed using FSL's FEAT ${ }^{3}$ by correlating the mean time series of the ROIs with the time series of all voxels in the brain in each child. The first-level language network connectivity maps between preterms and controls were contrasted with covariates of age and sex, and the group mean mixed effects Z (Gaussianized T/F statistics; Jenkinson and Woolrich, 2000) statistic images were thresholded using clusters determined by $Z>3.29$ and a corrected cluster significance threshold of FWE $p<0.05$ assuming a Gaussian random field for the $Z$-statistics (Worsley et al., 1992). In addition, children's neuropsychological characteristics, such verbal IQ (VIQ) scores, language outcomes (CL, RL, EL, LC, and LS), and gestational age were also explored in correlation with each subject's language network connectivity.

\section{RESULTS}

\section{Behavioral Measures}

Children born VPT showed significantly lower scores than FT controls in the PIQ $(t=-3.975, p<0.001)$, PSQ $(t=-3.656$, $p<0.001)$, and FSIQ $(t=-3.779, p<0.001)$ (Table 2). For VIQ, however, there was only a trend toward a difference between

${ }^{3}$ https://fsl.fmrib.ox.ac.uk/fsl/fslwiki/FEAT 
TABLE 2 | Neuropsychological assessments.

\begin{tabular}{|c|c|c|c|}
\hline & VPT (Mean \pm SD) & FT (Mean \pm SD) & $p$ \\
\hline \multicolumn{4}{|c|}{ Wechsler preschool and primary scales of intelligence - third edition (WPPSI-III) } \\
\hline Verbal IQ (VIQ) & $98.8 \pm 18.1$ & $106.7 \pm 11.6$ & $t=-1.919+$ \\
\hline Performance (PIQ) & $94.6 \pm 11.7$ & $107.5 \pm 11.7$ & $t=-3.975^{* * *}$ \\
\hline Processing speed (PSQ) & $92.2 \pm 18.7$ & $108.8 \pm 9.9$ & $t=-3.656^{* * *}$ \\
\hline Full-scale (FSIQ) & $94.0 \pm 14.2$ & $108.2 \pm 12.4$ & $t=-3.779^{* * *}$ \\
\hline \multicolumn{4}{|l|}{ (CELF Pre-2) } \\
\hline Core language score $(\mathrm{CL})$ & $95.31 \pm 16.10$ & $109.59 \pm 12.13$ & $t=-3.120^{* *}$ \\
\hline Receptive Language Index (RL) & $96.0 \pm 18.2$ & $116.6 \pm 7.7$ & $t=-4.547^{* * *}$ \\
\hline Expressive Language Index (EL) & $98.5 \pm 17.2$ & $115.3 \pm 10.5$ & $t=-2.849^{* *}$ \\
\hline Language contents Index (LC) & $98.4 \pm 16.7$ & $114.9 \pm 8.2$ & $t=-3.791^{* * *}$ \\
\hline Language Structure Index (LS) & $96.0 \pm 19.2$ & $117.5 \pm 10.9$ & $t=-3.294^{* *}$ \\
\hline \multicolumn{4}{|c|}{ Beery-Buktenica Developmental Test } \\
\hline Visual-motor integration (VMI) & $99.10 \pm 11.54$ & $110.48 \pm 8.93$ & $t=-3.788^{* * *}$ \\
\hline
\end{tabular}

the two groups and seemed partly attributable to the wide score range, from 66 to 133, in children born VPT $(t=-1.919$, $p<0.10)$. Children born VPT also scored significantly lower in all sub-scores of the CELF, as well as on the VMI (see Table 2).

\section{Group Differences in Primary Language Networks}

VPT children showed decreased functional connectivity overall in the primary language regions compared to FT controls (The group mean connectivity for each seed is presented in Supplement 2). Notably, connectivity from Broca's region (Broca L) did not show significant group differences, while the connectivity from other ROIs (Broca R, Wernicke L, and Wernicke R) was decreased in children born VPT compared with FT controls.

Compared to the FT group, VPT children showed significant reductions in connectivity between the Broca $\mathrm{R}$ to inferior frontal gyri (BA44), insulae (BA13), supramarginal gyri (BA40), and superior parietal lobules (BA7) bilaterally, as well as to the left posterior cingulate cortex (BA31) $(Z>3.29$, FWE $p<0.05$; Table 3 and Figure 1).

The Wernicke L connectivity to bilateral supramarginal gyri (BA40) and superior parietal lobule (BA7) was decreased in children born VPT, as well as the left angular gyrus (BA39) and the left posterior cingulate cortex (BA31) and the right postcentral gyrus (BA1) $(Z>3.29$, FWE $p<0.05$; Table 4 and Figure 2).

The decreased pattern of Wernicke R connectivity was seen only in contralateral connections in the VPT children, which were to the left supramarginal gyrus (BA40), left superior parietal lobule (BA7), posterior cingulate cortex (BA31), and precuneus (BA7) $(Z>3.29$, FWE $p<0.05$; Table 5 and Figure 3).

\section{Functional Connectivity Differences and Relations With Language Outcomes}

There were significant correlations between some behavioral outcomes and the language network that showed significant group differences. In all children, only the core language scores were positively correlated with Broca $\mathrm{R}$ connectivity, with areas including the right SMG cluster $(r=0.380, p=0.012)$, the left IFG cluster $(r=0.320, p=0.037)$, and the right IFG cluster $(r=0.315$, $p=0.040)$. In contrast, subjects' verbal IQ scores demonstrated no significant correlations with any connectivity values which showed group differences. The visual-motor integration scores were positively correlated with Broca $\mathrm{R}$ connectivity strength with the left SMG cluster $(r=0.386, p=0.005)$ and the right IFG cluster $(r=0.357, p=0.010)$.

We also examined if behavioral measures correlated with primary language networks at the whole brain level. The connectivity between primary language regions and motor/visual

TABLE 3 | Peak voxels with decreased functional connectivity from Broca's area homologue in children born VPT compared to full-term children.

\begin{tabular}{|c|c|c|c|c|c|c|c|}
\hline \multirow[t]{2}{*}{ Cluster } & \multirow[t]{2}{*}{ Peaks } & \multirow[t]{2}{*}{ L/R } & \multirow[t]{2}{*}{ BA } & \multicolumn{3}{|c|}{ MNI coordinates } & \multirow[t]{2}{*}{$z$} \\
\hline & & & & $x$ & $y$ & $z$ & \\
\hline \multirow[t]{4}{*}{1} & $\begin{array}{l}\text { Supramarginal } \\
\text { gyrus }\end{array}$ & $L$ & 40 & -60 & -34 & 42 & 4.68 \\
\hline & & $\mathrm{L}$ & 40 & -44 & -46 & 54 & 3.67 \\
\hline & & $L$ & 40 & -32 & -36 & 40 & 3.33 \\
\hline & $\begin{array}{l}\text { Posterior cingulate } \\
\text { cortex }\end{array}$ & $L$ & 31 & -14 & -26 & 40 & 3.64 \\
\hline \multirow[t]{3}{*}{2} & $\begin{array}{l}\text { Supramarginal } \\
\text { gyrus }\end{array}$ & $\mathrm{R}$ & 40 & 44 & -40 & 46 & 4.24 \\
\hline & $\begin{array}{l}\text { Inferior parietal } \\
\text { lobule }\end{array}$ & $\mathrm{R}$ & 7 & 22 & -64 & 44 & 3.67 \\
\hline & & $\mathrm{R}$ & 7 & 36 & -72 & 36 & 3.4 \\
\hline \multirow[t]{3}{*}{3} & Inferior frontal gyrus & $\mathrm{R}$ & 44 & 48 & 14 & 12 & 4.42 \\
\hline & & $R$ & 44 & 44 & 16 & 0 & 3.16 \\
\hline & Insula & $\mathrm{R}$ & 13 & 44 & 4 & 8 & 3.21 \\
\hline \multirow[t]{3}{*}{4} & Inferior frontal gyrus & $L$ & 44 & -48 & 4 & 8 & 3.52 \\
\hline & & & 44 & -44 & 2 & 26 & 3.37 \\
\hline & Insula & $\mathrm{L}$ & 13 & -42 & 10 & -14 & 3.42 \\
\hline
\end{tabular}

$L / R$, left hemisphere/right hemisphere; BA, Brodmann area; $Z>3.29$, FWE, $p<0.05$. 


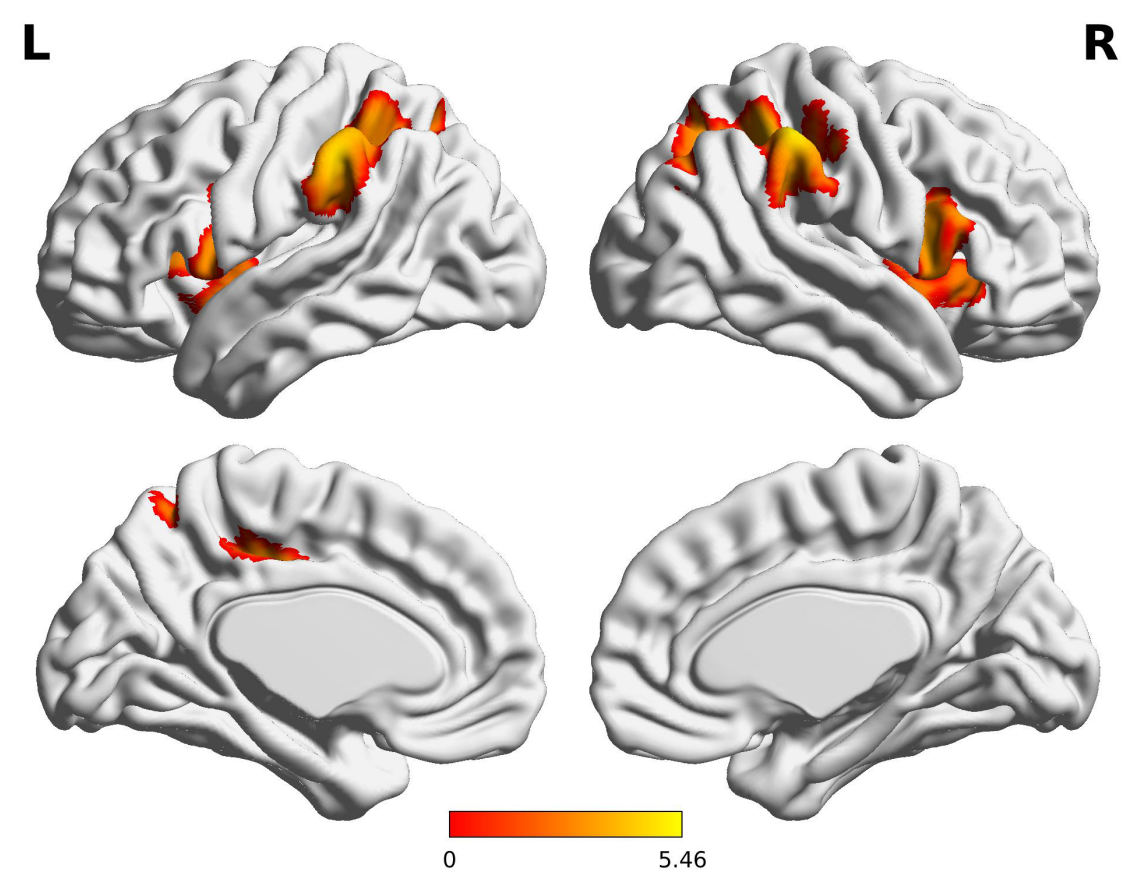

FIGURE 1 | Decreased functional connectivity from Broca's area homologue in children born VPT. Red-yellow regions show group differences of significantly decreased functional connectivity from Broca's area homologue in children born VPT.

regions were only correlated with core language scores (CL) for both groups. The Broca L connectivity with premotor cortex (BA6) and visual cortices (BA17 /BA19) were significantly correlated with $\mathrm{CL}$. The Broca $\mathrm{R}$ connectivity with premotor cortex (BA6) and postcentral gyrus (BA1) was also correlated with CL. In addition, Wernicke $\mathrm{L}$ but not $\mathrm{R}$ connectivity with visual area (BA18) was correlated with CL. When we examined the correlations between each language network and children's VIQ and GA, no significant correlations were found (see Supplement 3, 4 for more details).

TABLE 4 | Decreased functional connectivity from Wernicke's area in children born VPT, compared to full-term children.

\begin{tabular}{|c|c|c|c|c|c|c|c|}
\hline \multirow[t]{2}{*}{ Cluster } & \multirow[t]{2}{*}{ Peaks } & \multirow[t]{2}{*}{ L/R } & \multirow[t]{2}{*}{ BA } & \multicolumn{3}{|c|}{ MNI coordinates } & \multirow[t]{2}{*}{$z$} \\
\hline & & & & $x$ & $y$ & $z$ & \\
\hline \multirow[t]{4}{*}{1} & Supramarginal gyrus & L & 40 & -46 & -26 & 38 & 3.68 \\
\hline & & $L$ & 40 & -50 & -24 & 40 & 3.63 \\
\hline & & $\mathrm{L}$ & 40 & -34 & -36 & 44 & 3.41 \\
\hline & Angular gyrus & $\mathrm{L}$ & 39 & -50 & -54 & 46 & 3.28 \\
\hline \multirow[t]{6}{*}{2} & Postcentral gyrus & $\mathrm{R}$ & 1 & 48 & -24 & 40 & 3.98 \\
\hline & & $\mathrm{R}$ & 1 & 60 & -20 & 44 & 3.61 \\
\hline & & $\mathrm{R}$ & 1 & 54 & -26 & 54 & 3.43 \\
\hline & & $\mathrm{R}$ & 1 & 38 & -30 & 46 & 3.41 \\
\hline & Supramarginal gyrus & $\mathrm{R}$ & 40 & 58 & -36 & 58 & 3.75 \\
\hline & & $\mathrm{R}$ & 40 & 58 & -28 & 56 & 3.48 \\
\hline
\end{tabular}

L/R, left hemisphere/right hemisphere; $B A$, Brodmann area; $Z>3.29, F W E$ $p<0.05$.

\section{DISCUSSION}

The present study investigated language network connectivity in 4-year-old children born VPT and term-born controls born at term using defined language regions (Broca's and Wernicke's areas) and their homologues in the right hemisphere. We found significantly decreased functional connectivity in language networks in children born VPT compared to their term born peers, extending the previous literature in older children, which showed atypical functional connectivity in intrinsic language networks with decreased or increased patterns (Gozzo et al., 2009; Myers et al., 2010; Kwon et al., 2015), to this pre-school age range. A decrease in voxel-wise connectivity may reflect subtle injury or immaturity in regions of the brain resulting in vulnerability in the establishment of long-range connections (Kwon et al., 2015). Over time, injured or immature brain areas may also show increased patterns of connectivity, as the typically developing brain reduces connections between proximal regions or contralateral homologues to strengthen connections between remote regions by the pruning of the initial overabundance of synapses (Kesler et al., 2004; Ment and Vohr, 2008). Given these developmental trajectories, our findings support the model of atypical functional organization of intrinsic language network in children born very preterm in early childhood.

In our study, Broca's area homologue in the right hemisphere showed decreased connectivity to most primary language regions in children born VPT, with reduced connectivity to bilateral inferior frontal gyri (IFG) and bilateral supramarginal gyri (SMG). The atypical engagement of right-sided connectivity in the language network has been reported in children, 


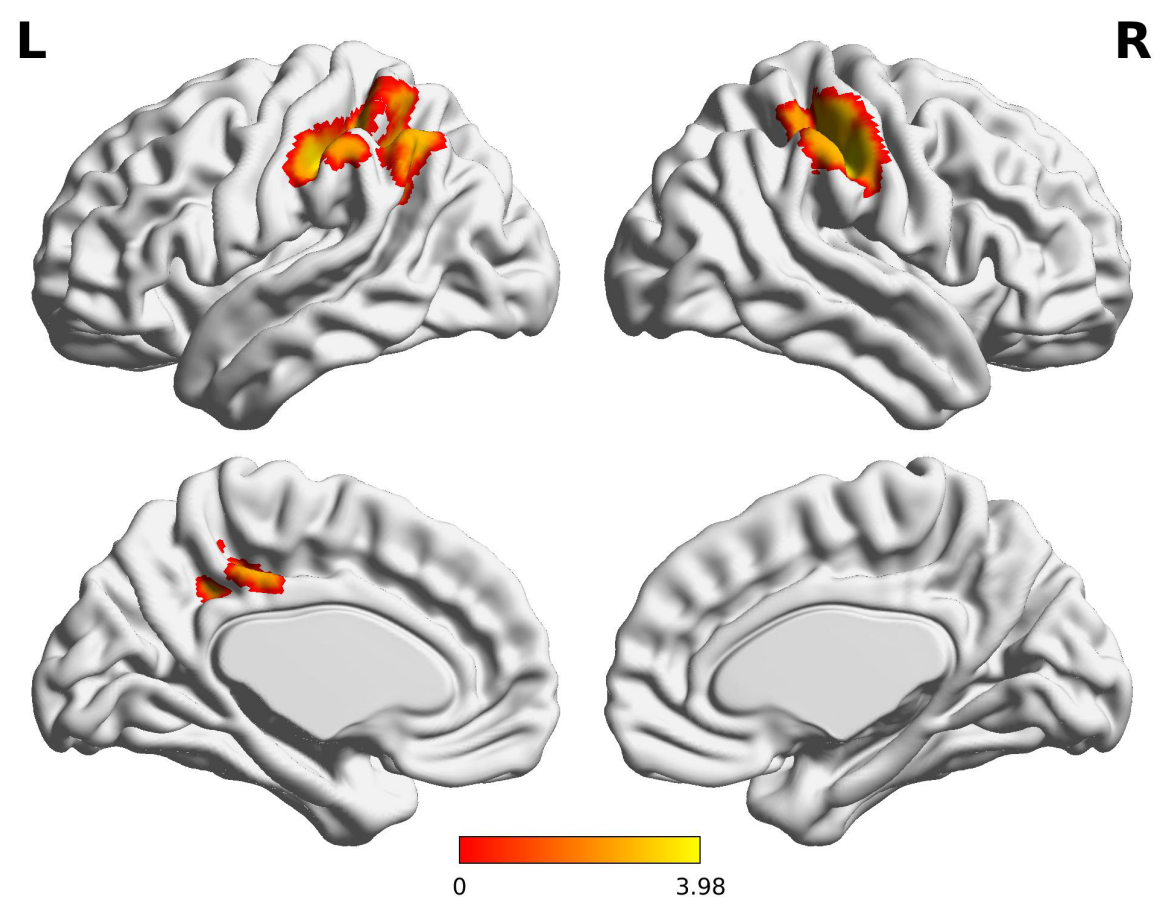

FIGURE 2 | Decreased functional connectivity from Wernicke's area in children born VPT. Red-yellow regions show group differences of significantly decreased functional connectivity from Wernicke's area in children born VPT.

adolescents, and young adults born preterm, suggesting that preterm birth affects developmental functional lateralization in the brain (Gozzo et al., 2009; Myers et al., 2010; Friederici et al., 2011; Scheinost et al., 2014). Given that the leftright asymmetry of the developing brain is mediated by programmed gene expression during the second and third trimester of gestation (Sun et al., 2005), preterm birth may alter this neurogenesis (Vaccarino and Ment, 2004; Malik et al., 2013) and result in long-lasting changes in functional organization. It is not clear, however, whether this pattern of connectivity represents a delay in cerebral maturation or a compensatory mechanism to overcome difficulties in language processing (Shaywitz et al., 2002; Rushe et al., 2004; Chilosi et al., 2005; Gozzo et al., 2009). Decreased connectivity from Broca's right homologue in our study may reflect functional immaturities of the very preterm children at an early stage

TABLE 5 | Decreased functional connectivity from Wernicke's area homologue in children born VPT, compared to full-term children.

\begin{tabular}{|c|c|c|c|c|c|c|c|}
\hline \multirow[t]{2}{*}{ Cluster } & \multirow[t]{2}{*}{ Peaks } & \multirow[t]{2}{*}{ L/R } & \multirow[t]{2}{*}{ BA } & \multicolumn{3}{|c|}{ MNI coordinates } & \multirow[t]{2}{*}{$z$} \\
\hline & & & & $x$ & $y$ & $z$ & \\
\hline \multirow[t]{2}{*}{1} & $\begin{array}{l}\text { Posterior cingulate } \\
\text { cortex }\end{array}$ & L & 31 & -14 & -44 & 38 & 3.33 \\
\hline & $\begin{array}{l}\text { Inferior parietal } \\
\text { lobule }\end{array}$ & L & 1 & -34 & -32 & 40 & 3.07 \\
\hline
\end{tabular}

L/R, left hemisphere/right hemisphere; $B A$, Brodmann area; $Z>3.29, F W E$ $p<0.05$. of language development, as cerebral language lateralization, dominant in left inferior frontal cortex and supporting topdown processing of language, is not yet fully specialized at this age (Skeide and Friederici, 2016). A compensatory strategy of right-sided language network in the preterm population could emerge gradually coupled with leftward lateralization and the development of top-down processing in later development.

Broca's homologue in the right hemisphere showed significantly decreased inter-hemispheric and intra-hemispheric connections with bilateral IFG and SMG in the VPT group. There is evidence of strong inter-hemispheric functional connectivity between left and right IFG in typically developing children aged 3 to 5 years with a weakening pattern with increasing age (Xiao et al., 2016a). Six-year-old children still showed stronger interhemispheric connectivity compared to adults (Friederici et al., 2011). In children aged 5-6 years, the strong inter-hemispheric connection was correlated with more advanced language abilities (Xiao et al., 2016b). This strong inter-hemispheric correlation in typical development of the language network is partly supported by structural maturation of the splenium, through which the auditory commissures project and the interplay of left and right hemispheric language functions is possible (Dubois et al., 2008; Lebel et al., 2008). Thus, our findings of decreased inter-hemispheric connections from Broca's homologue to left IFG indicates that preterm children are deviating from typical development at an early stage of life, and may be related to other findings of reductions in structural connectivity measures (Young et al., 2018). In addition, the decreased intra-hemispheric 


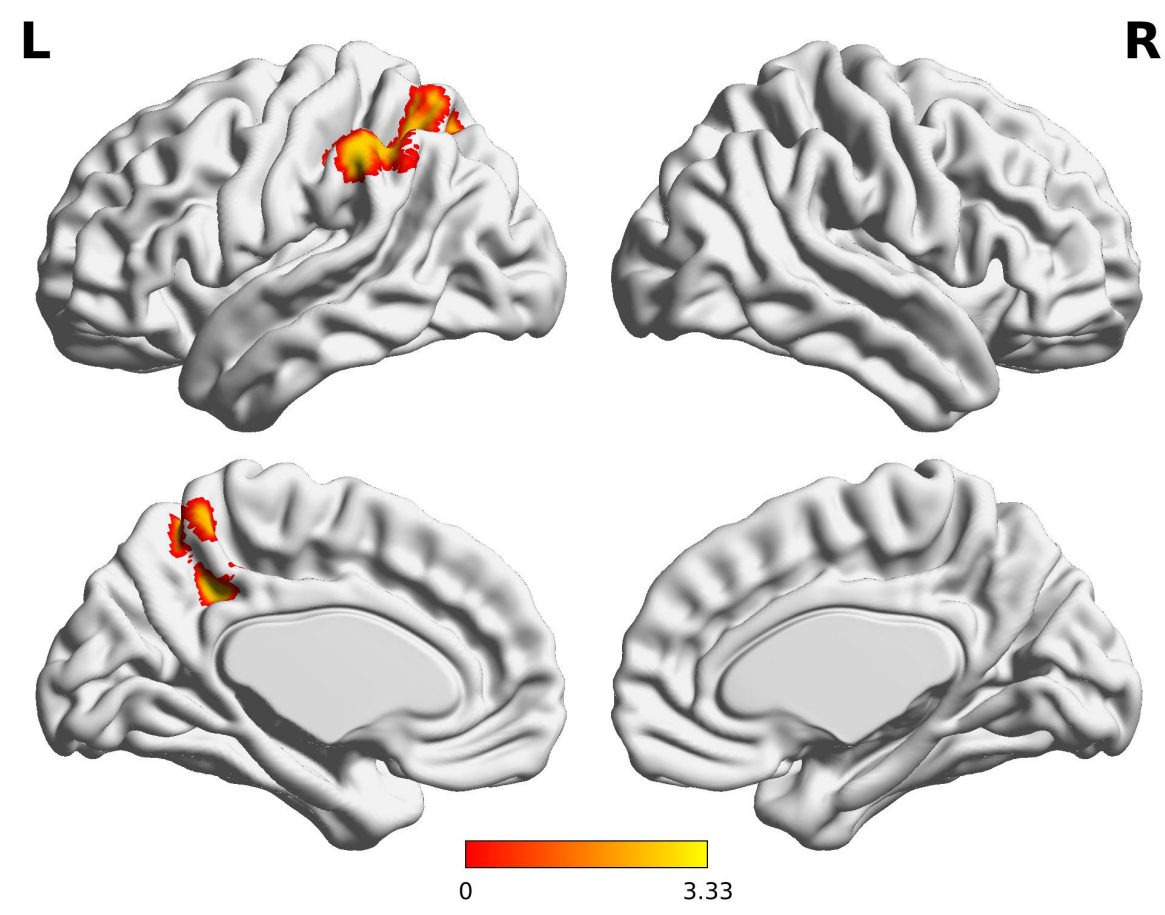

FIGURE 3 | Decreased functional connectivity from Wernicke's area homologue in children born VPT. Red-yellow regions show group differences of significantly decreased functional connectivity from Wernicke's area homologue in children born VPT.

connectivity from Broca's homologue to the right IFG and SMG may be further evidence of atypical language networks in children born VPT.

Typically developing children demonstrate a more right-sided functional lateralization during language processing compared to adults (Brauer and Friederici, 2007), and this may be related to children's higher reliance on prosodic information during language processing, supported by the right hemisphere (Wartenburger et al., 2007; Sabisch et al., 2009). Even in the mature brain, suprasegmental prosodic information tends to be processed in the right superior temporal gyrus and the right inferior frontal gyrus. The right-dominant activation for phonological processing is also reported in young children (Sugiura et al., 2011). Given behavioral evidence that children born prematurely are at higher risk for delayed or atypical processing of phonetic and prosodic information (GonzalezGomez and Nazzi, 2012; Ragó et al., 2014), decreased intrahemispheric connection in the right hemisphere in children born VPT may underlie these specific deficits in their language development.

Both Wernicke's area and its right hemisphere homologue also showed decreased inter-hemispheric connectivity to bilateral SMG in the VPT group, which represented alterations in shorter-range connectivity, compared to Broca's area homologues which showed alterations in long range connectivity to bilateral SMG. In addition, Wernicke's area also showed decreased intrahemispheric connectivity to the left SMG and angular gyrus (AG). The bottom-up processes for cortical language networks, which involve low-level computation of mental representations from the sensory input, primarily implemented bilaterally in the temporal cortices, develop rapidly in first 3 years of life (Skeide and Friederici, 2016), and strong inter-hemispheric connections in these areas are observed from birth to early childhood. By 3 years of age, in typically developing children the intrinsic language network from the left posterior superior temporal gyrus is functionally correlated with bilateral inferior parietal lobe, left superior parietal lobe, and angular gyrus (Xiao et al., 2016a). At 6 years of age, children's language network is still characterized by a strong functional inter-hemispheric connectivity, mainly between the superior temporal regions, compared to adults (Friederici et al., 2011). Given this typical developmental trajectory, preterm birth appears to disturb the development of bottom-up language processing network; these difficulties extend to older children and adolescents with reports of atypical connectivity between Wernicke's area and SMG (Gozzo et al., 2009; Myers et al., 2010; van Ettinger-Veenstra et al., 2017).

Notably, the decreased connectivity from primary language regions in children born VPT consistently included the left SMG, which is widely accepted as a part of Wernicke's area (Binder, 2015) and has traditionally been thought to be responsible for phonologic processing (Ment et al., 2006). This is also concordant with findings that reported atypical involvement of the left SMG with Broca's area homologue in preterm children at schoolage (Gozzo et al., 2009). Taken together, our results support the model that children born VPT have significant decreases in neural networks that support auditory phonologic processing of language. 
The connectivity strength of Broca's area homologue with primary language regions was positively correlated with children's language outcomes. The better the language outcomes at 4 years of age, the higher the connectivity strength between Broca's area homologue and the right SMG as well as between Broca's area homologue and the left IFG. Interestingly, the primary language network connectivity at 4 years of age was associated with the core language score and the visual-motor integration score, which may reflect that the language network organization in the early childhood is related to general language ability as well as the coordination with sensory information processing and motor skills. The evidence of decreased connectivity in Broca's area homologue in children born VPT in our study also points to the critical delays happening in this population.

There are several limitations to our study. A relatively small sample size may have restricted the generalizability of our findings to understanding children born very preterm. We included age as a covariate in our analyses since the term-born children were older than children born very preterm by a few months. We expect exactly age-matched controls in future studies would provide higher statistical power. Also most of our VPT sample showed only mild cognitive delays compared to their term-born peers. Future studies would require larger samples of children, and with a wider range of outcomes. Lastly, $5 \mathrm{~min}$ for rs-fMRI scans was a long time for our participants, as they were a very young clinical population, but we acknowledge that more volumes would be an advantage for analyses and stronger statistical power.

\section{CONCLUSION}

In conclusion, we found atypical functional organization underlying language deficits in 4-year-old children born VPT, who showed decreased intra- and inter-hemispheric connectivity among primary language regions compared to their term-born peers. Broca's area homologue in the right hemisphere showed

\section{REFERENCES}

Aarnoudse-Moens, C. S., Duivenvoorden, H. J., Weisglas-Kuperus, N., Van Goudoever, J. B., and Oosterlaan, J. (2012). The profile of executive function in very preterm children at 4 to 12 years. Dev. Med. Child Neurol. 54, 247-253. doi: 10.1111/j.1469-8749.2011.04150.x

Barre, N., Morgan, A., Doyle, L. W., and Anderson, P. J. (2011). Language abilities in children who were very preterm and/or very low birth weight: a meta-analysis. J. Pediatr. 158, 766-774. doi: 10.1016/j.jpeds.2010.10.032

Bäuml, J. G., Daamen, M., Meng, C., Neitzel, J., Scheef, L., Jaekel, J., et al. (2014). Correspondence between aberrant intrinsic network connectivity and graymatter volume in the ventral brain of preterm born adults. Cereb. Cortex 25, 4135-4145. doi: 10.1093/cercor/bhu133

Beery, K. E., Buktenica, N. A., and Beery, N. A. (2004). The Beery-Buktenica Developmental Test of Visual-Motor Integration, 5th Edn. Minneapolis, MN: NCS Pearson.

Berk, L. E. (2013). Child Development, 9th Edn. Boston: Pearson.

Binder, J. R. (2015). The wernicke area modern evidence and a reinterpretation. Neurology 85, 2170-2175. doi: 10.1212/WNL.0000000000002219 the most marked functional connectivity effects, suggesting that preterm birth may cause fundamental alterations in the cerebral lateralization for language. Our findings demonstrate that altered functional connectivity in the language network in children born VPT is already present in the preschool age period and may represent neural bases of consistent lower language outcomes in this population.

\section{AUTHOR CONTRIBUTIONS}

MT and JY contributed to designing the study and collecting the data. EC and MV analyzed the data. EC, MV, and MT interpreted the results. EC wrote a draft of the manuscript. MV, JY, and MT reviewed and revised the manuscript. All authors confirmed the final version of the manuscript.

\section{FUNDING}

The acquisition of these data was funded by Canadian Institutes of Health Research grants to MT (MOP-84399 and MOP137115).

\section{ACKNOWLEDGMENTS}

We would like to thank all of the families who participated in the study. We also thank Sarah Mossad and MyLoi Huynh-Silveira for their significant support and contributions and Tammy Rayner and Ruth Weiss for all their invaluable help with MRI acquisitions.

\section{SUPPLEMENTARY MATERIAL}

The Supplementary Material for this article can be found online at: https://www.frontiersin.org/articles/10.3389/fnhum. 2018.00512/full\#supplementary-material

Brauer, J., and Friederici, A. D. (2007). Functional neural networks of semantic and syntactic processes in the developing brain. J. Cogn. Neurosci. 19, 1609-1623. doi: 10.1162/jocn.2007.19.10.1609

Carreiras, M., Seghier, M. L., Baquero, S., Estévez, A., Lozano, A., Devlin, J. T., et al. (2009). An anatomical signature for literacy. Nature 461:983. doi: 10.1038/ nature08461

Chilosi, A. M., Pecini, C., Cipriani, P., Brovedani, P., Brizzolara, D., Ferretti, G., et al. (2005). Atypical language lateralization and early linguistic development in children with focal brain lesions. Dev. Med. Child Neurol. 47, 725-730. doi: 10.1017/S0012162205001532

Constable, R. T., Vohr, B. R., Scheinost, D., Benjamin, J. R., Fulbright, R. K., Lacadie, C., et al. (2013). A left cerebellar pathway mediates language in prematurely-born young adults. Neuroimage 64, 371-378. doi: 10.1016/j. neuroimage.2012.09.008

Counsell, S. J., Rutherford, M. A., Cowan, F. M., and Edwards, A. D. (2003). Magnetic resonance imaging of preterm brain injury. Arch. Dis. Childhood-Fetal Neonat. Edn. 88, F269-F274. doi: 10.1136/fn.88.4.F269

Dubois, J., Dehaene-Lambertz, G., Perrin, M., Mangin, J. F., Cointepas, Y., Duchesnay, E., et al. (2008). Asynchrony of the early maturation of white matter 
bundles in healthy infants: quantitative landmarks revealed noninvasively by diffusion tensor imaging. Hum. Brain Mapp. 29, 14-27. doi: 10.1002/hbm.20363

Foster-Cohen, S., Edgin, J. O., Champion, P. R., and Woodward, L. J. (2007). Early delayed language development in very preterm infants: evidence from the MacArthur-Bates CDI. J. Child Lang. 34, 655-675. doi: 10.1017/ S0305000907008070

Friederici, A. D., Brauer, J., and Lohmann, G. (2011). Maturation of the language network: from inter-to intrahemispheric connectivities. PLoS One 6:e20726. doi: 10.1371/journal.pone.0020726

Friston, K. J., Frith, C. D., Fletcher, P., Liddle, P. F., and Frackowiak, R. S. (1996). Functional topography: multidimensional scaling and functional connectivity in the brain. Cereb. Cortex 6, 156-164. doi: 10.1093/cercor/6.2.156

Gonzalez-Gomez, N., and Nazzi, T. (2012). Phonotactic acquisition in healthy preterm infants. Dev. Sci. 15, 885-894. doi: 10.1111/j.1467-7687.2012.01186.x

Gozzo, Y., Vohr, B., Lacadie, C., Hampson, M., Katz, K. H., Maller-Kesselman, J., et al. (2009). Alterations in neural connectivity in preterm children at school age. Neuroimage 48, 458-463. doi: 10.1016/j.neuroimage.2009.06.046

Isaacs, E. B., Edmonds, C. J., Chong, W. K., Lucas, A., Morley, R., and Gadian, D. G. (2004). Brain morphometry and IQ measurements in preterm children. Brain 127, 2595-2607. doi: 10.1093/brain/awh300

Jenkinson, M., and Woolrich, M. (2000). Asymptotic T to $Z$ and F to Z Statistic Transformations. Internal Technical Report: TR00MJ1. Oxford: Oxford Centre for Functional Magnetic Resonance Imaging of the Brain, Department of Clinical Neurology, Oxford University.

Kesler, S. R., Ment, L. R., Vohr, B., Pajot, S. K., Schneider, K. C., Katz, K. H., et al. (2004). Volumetric analysis of regional cerebral development in preterm children. Pediatr. Neurol. 31, 318-325. doi: 10.1016/j.pediatrneurol.2004. 06.008

Kwon, S. H., Scheinost, D., Lacadie, C., Sze, G., Schneider, K. C., Dai, F., et al. (2015). Adaptive mechanisms of developing brain: cerebral lateralization in the prematurely-born. Neuroimage 108, 144-150. doi: 10.1016/j.neuroimage.2014. 12.032

Kwon, S. H., Scheinost, D., Vohr, B., Lacadie, C., Schneider, K., Dai, F., et al. (2016). Functional magnetic resonance connectivity studies in infants born preterm: suggestions of proximate and long-lasting changes in language organization. Dev. Med. Child Neurol. 58, 28-34. doi: 10.1111/dmcn.13043

Lebel, C., Walker, L., Leemans, A., Phillips, L., and Beaulieu, C. (2008). Microstructural maturation of the human brain from childhood to adulthood. Neuroimage 40, 1044-1055. doi: 10.1016/j.neuroimage.2007.12.053

Lind, A., Parkkola, R., Lehtonen, L., Munck, P., Maunu, J., and Lapinleimu, H. (2011). Associations between regional brain volumes at term-equivalent age and development at 2 years of age in preterm children. Pediatr. Radiol. 41, 953-961. doi: 10.1007/s00247-011-2071-X

Luu, T. M., Vohr, B. R., Schneider, K. C., Katz, K. H., Tucker, R., Allan, W. C., et al. (2009). Trajectories of receptive language development from 3 to 12 years of age for very preterm children. Pediatrics 124, 333-341. doi: 10.1542/peds.2008-2587

Malik, S., Vinukonda, G., Vose, L. R., Diamond, D., Bhimavarapu, B. B., Hu, F., et al. (2013). Neurogenesis continues in the third trimester of pregnancy and is suppressed by premature birth. J. Neurosci. 33, 411-423. doi: 10.1523/ JNEUROSCI.4445-12.2013

Manuck, T. A., Rice, M. M., Bailit, J. L., Grobman, W. A., Reddy, U. M., Wapner, R. J., et al. (2016). Preterm neonatal morbidity and mortality by gestational age: a contemporary cohort. Am. J. Obstet. Gynecol. 215, 103-e1. doi: 10.1016/j.ajog. 2016.01.004

Ment, L. R., Kesler, S., Vohr, B., Katz, K. H., Baumgartner, H., Schneider, K. C., et al. (2009). Longitudinal brain volume changes in preterm and term control subjects during late childhood and adolescence. Pediatrics 123, 503-511. doi: 10.1542/peds.2008-0025

Ment, L. R., Peterson, B. S., Meltzer, J. A., Vohr, B., Allan, W., Katz, K. H., et al. (2006). A functional magnetic resonance imaging study of the long-term influences of early indomethacin exposure on language processing in the brains of prematurely born children. Pediatrics 118, 961-970. doi: 10.1542/peds.20052870

Ment, L. R., and Vohr, B. R. (2008). Preterm birth and the developing brain. Lancet Neurol. 7:378. doi: 10.1016/S1474-4422(08)70073-5

Myers, E. H., Hampson, M., Vohr, B., Lacadie, C., Frost, S. J., Pugh, K. R., et al. (2010). Functional connectivity to a right hemisphere language center in prematurely born adolescents. Neuroimage 51, 1445-1452. doi: 10.1016/j. neuroimage.2010.03.049

Nosarti, C., Mechelli, A., Herrera, A., Walshe, M., Shergill, S. S., Murray, R. M., et al. (2011). Structural covariance in the cortex of very preterm adolescents: a voxel-based morphometry study. Hum. Brain Mapp. 32, 1615-1625. doi: 10.1002/hbm.21133

Parker, J., Mitchell, A., Kalpakidou, A., Walshe, M., Jung, H. Y., Nosarti, C., et al. (2008). Cerebellar growth and behavioural \& neuropsychological outcome in preterm adolescents. Brain 131, 1344-1351. doi: 10.1093/brain/awn062

Peña, M., Pittaluga, E., and Mehler, J. (2010). Language acquisition in premature and full-term infants. Proc. Natl. Acad. Sci. U.S.A. 107, 3823-3828. doi: 10.1073/ pnas.0914326107

Peña, M., Werker, J. F., and Dehaene-Lambertz, G. (2012). Earlier speech exposure does not accelerate speech acquisition. J. Neurosci. 32, 11159-11163. doi: 10. 1523/JNEUROSCI.6516-11.2012

Putnick, D. L., Bornstein, M. H., Eryigit-Madzwamuse, S., and Wolke, D. (2017). Long-term stability of language performance in very preterm, moderate-late preterm, and term children. J. Pediatr. 181, 74-79. doi: 10.1016/j.jpeds.2016. 09.006

Ragó, A., Honbolygó, F., Róna, Z., Beke, A., and Csépe, V. (2014). Effect of maturation on suprasegmental speech processing in full-and preterm infants: a mismatch negativity study. Res. Dev. Disabil. 35, 192-202. doi: 10.1016/j.ridd. 2013.10.006

Rushe, T. M., Temple, C. M., Rifkin, L., Woodruff, P. W. R., Bullmore, E. T., Stewart, A. L., et al. (2004). Lateralisation of language function in young adults born very preterm. Arch. Dis. Childhood-Fetal Neonat. Edn. 89, F112-F118. doi: 10.1136/adc.2001.005314

Sabisch, B., Hahne, C. A., Glass, E., von Suchodoletz, W., and Friederici, A. D. (2009). Children with specific language impairment: the role of prosodic processes in explaining difficulties in processing syntactic information. Brain Res. 1261, 37-44. doi: 10.1016/j.brainres.2009.01.012

Sansavini, A., Guarini, A., Justice, L. M., Savini, S., Broccoli, S., Alessandroni, R., et al. (2010). Does preterm birth increase a child's risk for language impairment? Early Hum. Dev. 86, 765-772. doi: 10.1016/j.earlhumdev.2010. 08.014

Scheinost, D., Benjamin, J., Lacadie, C. M., Vohr, B., Schneider, K. C., Ment, L. R., et al. (2012). The intrinsic connectivity distribution: a novel contrast measure reflecting voxel level functional connectivity. Neuroimage 62, 1510-1519. doi: 10.1016/j.neuroimage.2012.05.073

Scheinost, D., Lacadie, C., Vohr, B. R., Schneider, K. C., Papademetris, X., Constable, R. T., et al. (2014). Cerebral lateralization is protective in the very prematurely born. Cereb. Cortex 25, 1858-1866. doi: 10.1093/cercor/ bht 430

Shaywitz, B. A., Shaywitz, S. E., Pugh, K. R., Mencl, W. E., Fulbright, R. K., Skudlarski, P., et al. (2002). Disruption of posterior brain systems for reading in children with developmental dyslexia. Biol. Psychiatry 52, 101-110. doi: 10. 1016/S0006-3223(02)01365-3

Skeide, M. A., and Friederici, A. D. (2016). The ontogeny of the cortical language network. Nat. Rev. Neurosci. 17:323. doi: 10.1038/nrn.2016.23

Smyser, C. D., Snyder, A. Z., Shimony, J. S., Mitra, A., Inder, T. E., and Neil, J. J. (2014). Resting-state network complexity and magnitude are reduced in prematurely born infants. Cereb. Cortex 26, 322-333. doi: 10.1093/cercor/ bhu251

Sugiura, L., Ojima, S., Matsuba-Kurita, H., Dan, I., Tsuzuki, D., Katura, T., et al. (2011). Sound to language: different cortical processing for first and second languages in elementary school children as revealed by a large-scale study using fNIRS. Cereb. Cortex 21, 2374-2393. doi: 10.1093/cercor/bhr023

Sun, T., Patoine, C., Abu-Khalil, A., Visvader, J., Sum, E., Cherry, T. J., et al. (2005). Early asymmetry of gene transcription in embryonic human left and right cerebral cortex. Science 308, 1794-1798. doi: 10.1126/science.11 10324

Thomason, M. E., Scheinost, D., Manning, J. H., Grove, L. E., Hect, J., Marshall, N., et al. (2017). Weak functional connectivity in the human fetal brain prior to preterm birth. Sci. Rep. 7:39286. doi: 10.1038/srep39286

Tomasi, D., and Volkow, N. D. (2012). Resting functional connectivity of language networks: characterization and reproducibility. Mol. Psychiatry 17, 841-854. doi: $10.1038 / \mathrm{mp} .2011 .177$ 
Vaccarino, F. M., and Ment, L. R. (2004). Injury and repair in developing brain. Arch. Dis. Childhood-Fetal Neonat. Edn. 89, F190-F192. doi: 10.1136/adc.2003. 043661

van Ettinger-Veenstra, H., Widén, C., Engström, M., Karlsson, T., Leijon, I., and Nelson, N. (2017). Neuroimaging of decoding and language comprehension in young very low birth weight (VLBW) adolescents: indications for compensatory mechanisms. PLoS One 12:e0185571. doi: 10.1371/journal.pone.01 85571

van Noort-van der Spek, I. L., Franken, M. C. J., and Weisglas-Kuperus, N. (2012). Language functions in preterm-born children: a systematic review and meta-analysis. Pediatrics 129, 745-754. doi: 10.1542/peds.20111728

Vohr, B. (2014). Speech and language outcomes of very preterm infants. Semin. Fetal Neonat. Med. 19, 78-83. doi: 10.1016/j.siny.2013. 10.007

Wartenburger, I., Steinbrink, J., Telkemeyer, S., Friedrich, M., Friederici, A. D., and Obrig, H. (2007). The processing of prosody: evidence of interhemispheric specialization at the age of four. Neuroimage 34, 416-425. doi: 10.1016/j. neuroimage.2006.09.009

Wechsler, D. (2002). WPPSI-III Administration and Scoring Manual. San Antonio, TX: Psychological Corporation.

Wiig, E. H., Secord, W., and Semel, E. M. (2004). CELF Preschool 2: Clinical Evaluation of Language Fundamentals Preschool. San Antonio, TX: Pearson/PsychCorp.

World Health Organization [WHO], (2018). WHO Media Center Report. Available at: http://www.who.int/mediacentre/factsheets/fs363/en

Xiao, Y., Brauer, J., Lauckner, M., Zhai, H., Jia, F., Margulies, D. S., et al. (2016a). Development of the intrinsic language network in preschool children from ages 3 to 5 years. PLoS One 11:e0165802. doi: 10.1371/journal.pone. 0165802
Worsley, K. J., Evans, A. C., Marrett, S., and Neelin, P. (1992). A three-dimensional statistical analysis for CBF activation studies in human brain. J. Cereb. Blood Flow Metab. 12, 900-918. doi: 10.1038/jcbfm.1992.127

Xiao, Y., Friederici, A. D., Margulies, D. S., and Brauer, J. (2016b). Longitudinal changes in resting-state fMRI from age 5 to age 6years covary with language development. Neuroimage 128, 116-124. doi: 10.1016/j.neuroimage.2015. 12.008

Young, J. M., Powell, T. L., Morgan, B. R., Card, D., Lee, W., Smith, M. L., et al. (2015). Deep grey matter growth predicts neurodevelopmental outcomes in very preterm children. Neuroimage 111, 360-368. doi: 10.1016/j.neuroimage. 2015.02.030

Young, J. M., Vandewouw, M. M., Morgan, B. R., Smith, M. L., Sled, J. G., and Taylor, M. J. (2018). Altered white matter development in children born very preterm. Brain Funct. Struct. 223, 2129-2141. doi: 10.1371/journal.pone. 0076702

Zhu, L., Fan, Y., Zou, Q., Wang, J., Gao, J. H., and Niu, Z. (2014). Temporal reliability and lateralization of the resting-state language network. PLoS One 9:e85880. doi: 10.1371/journal.pone.0085880

Conflict of Interest Statement: The authors declare that the research was conducted in the absence of any commercial or financial relationships that could be construed as a potential conflict of interest.

Copyright (C) 2018 Choi, Vandewouw, Young and Taylor. This is an open-access article distributed under the terms of the Creative Commons Attribution License (CC BY). The use, distribution or reproduction in other forums is permitted, provided the original author(s) and the copyright owner(s) are credited and that the original publication in this journal is cited, in accordance with accepted academic practice. No use, distribution or reproduction is permitted which does not comply with these terms. 